\title{
Caracterización de la respuesta de las comunidades locales a la influencia directa de la variabilidad climática en el Caribe Sur de Costa Rica
}

\section{Characterization of the response of local communities to the direct effects of climate variability on the South Caribbean coast of Costa Rica}

\author{
Lilliana Piedra Castro ${ }^{1}$ \\ Universidad Nacional, Costa Rica \\ Aida Caridad Hernández Zanuy²
Universidad de La Habana, Cuba
}

\author{
Luis Manuel Sierra Sierra ${ }^{3}$ \\ José Miguel Pereira Chávez ${ }^{4}$ \\ Maikol Castillo Chinchilla ${ }^{5}$ \\ Vanessa Morales Cerdas ${ }^{6}$ \\ Universidad Nacional, Costa Rica
}

\begin{abstract}
Resumen. La variabilidad climática provoca impactos sobre los ecosistemas marino-costeros, además de la afectación de los recursos naturales, paisajes y las comunidades costeras vinculadas. Tales comunidades pueden adaptarse a estos cambios o convertirse en migrantes climáticos. Este estudio tuvo como propósito evaluar los mecanismos de adaptación de las comunidades costeras del Caribe Sur (CS) de Costa Rica en relación con los impactos de la variabilidad climática sobre los ecosistemas marinos y costeros para la reducción de su vulnerabilidad. Se realizó revisión documental, se aplicaron técnicas de observación no participante y encuestas a pobladores locales. Se realizaron 65 encuestas en las localidades de Gandoca, Manzanillo, Cahuita, Puerto Viejo, del cantón de Talamanca y Limón del cantón de Limón. Entre los principales resultados destacan las actividades económicas registradas, que fueron agricultura de subsistencia, pesca, turismo, comercio y recolección de cocos. Las artes de pesca identificadas fueron buceo a pulmón, caña de pescar, nasas, líneas de palangre y trasmallo. Se identificó que los locales perciben la influencia de la variabilidad climática; por ejemplo, el aumento del nivel del mar debido a que ha afectado la infraestructura (casas, carreteras, puentes) y el aumento de la temperatura atmosférica porque ha impactado la distribución de las especies de peces. Las comunidades locales no cuentan con un plan de acción gubernamental; pero se han adaptado a las nuevas condiciones, implementado acciones como: diversificación del voluntariado, traslado de infraestructura, agricultura orgánica, pesca como actividad económica complementaria y reforestación de la línea costera. Estas comunidades se han adaptado a los cambios presentados por la variabilidad climática, aunque sin planificación, por lo que es necesaria la atención urgente de las autoridades para definir acciones en el futuro.
\end{abstract}

Palabras clave. Caribe Sur, Costa Rica, comunidades humanas, adaptación, variabilidad climática

\begin{abstract}
The climatic variability causes impacts on the marine-coastal ecosystems, implies the affectation of the natural resources, landscapes and the coastal communities linked. These communities can adapt to these changes or become climatic migrants. This study aimed to evaluate the adaptation mechanisms of the coastal communities of the Southern Caribbean (SC) of Coast to the impacts of climatic variability on the marine and coastal ecosystems for the reduction of their vulnerability. A literature review was carried out, non-participant observation techniques and surveys of local people were applied. 65 surveys were conducted in five communities. The main economic activities were subsistence agriculture, fishing, tourism, commerce and collection of coconuts. The fishing gear used is scuba diving, fishing rod, pots, longline and trammel lines. The influence of climate variability was identified; For example, the rise in sea level has affected infrastructure (houses, roads, bridges) and the increase in atmospheric temperature has impacted the distribution of fish species. Local communities do not have a government action plan, but have adapted to the new conditions, implementing actions such as: diversification of volunteering, transfer of infrastructure, organic agriculture, fishing as a complementary economic activity and reforestation of the coastline. These communities have adapted to the changes presented by climate variability, but without planning, so urgent attention is needed from the authorities to define future actions.
\end{abstract}

Keywords. South Caribbean, Costa Rica, human communities, adaptation, climate change

\footnotetext{
${ }^{1}$ Doctora en Ciencias Naturales para el Desarrollo. Laboratorio de Recursos Naturales y Vida Silvestre (LARNAVISI), Escuela de Ciencias Biológicas, Universidad Nacional, Costa Rica. Dirección electrónica: lilliana.piedra.castro@una.cr

${ }^{2}$ Doctora en Ciencias Biológicas, Instituto de Ciencias del Mar, Universidad de la Habana, Cuba. Dirección electrónica: ahernandezzanuy@gmail.com

3Doctor en Ciencias Biológicas. Universidad Nacional, Costa Rica. Dirección electrónica: Imsierra1@yahoo.com.mx

${ }^{4}$ Doctor en Educación. Universidad Nacional, Costa Rica. Dirección electrónica: jose.pereira.chaves@una.cr

${ }^{5}$ Master en Sistemas de Información Geográfica y Teledetección. Universidad Nacional, Costa Rica. Dirección electrónica: maikol.castillo.chinchilla@una.cr

6Licenciada en Manejo de Recursos Naturales. Universidad Nacional, Costa Rica. Dirección electrónica: vanessa.morales.cerdas@una.cr
} 


\section{Introducción}

La variabilidad climática es un fenómeno sobre el cual se han pronosticado diversos efectos e influencias en la biosfera, los más relevantes incidirán sobre los ecosistemas naturales y especialmente, los marinos y costeros (Rojas, Campos, Alpízar, Bravo-Chacón y CórdobaMuñoz, 2003; Anadón, Duarte y Fariña, 2005; Carballo, Bautista-Guerrero, Nava y CruzBarraza, 2010; Yáñez-Arancibia, Twilley y LaraDomínguez, 2016).

Entre los efectos que se esperan sobre la zona marina se encuentran la reducción de la productividad primaria, los cambios en la distribución del plancton (fito y zooplancton) y hasta de los vertebrados mayores, así como de las redes tróficas, mortalidad de organismos por cambios en la temperatura y modificaciones de las características físicas y químicas de las masas de agua. También, se espera mayor virulencia de parásitos e impacto sobre las poblaciones nativas debido a la presencia de las especies invasoras (De la Rosa y Zazo, 2005; Sharp, 2004).

En la zona costera, se plantea que se presentarán efectos importantes entre los que destaca la erosión de la línea de costa, cambios en la ubicación de la línea de costa y en la vegetación costera, salinización de los cuerpos de agua dulce, pérdida de infraestructura ubicada en la zona marítima, entre otros (Felicísimo, Muñóz, Mateo y Villalba, 2012; Hernández, Ortiz, Méndez y Gama, 2008; Yáñez-Arancibia, Twilley y Lara-Domínguez, 2016).

Adicionalmente, los impactos que la variabilidad climática alcanza a las comunidades costeras, y pueden ser considerados especialmente graves. Por ejemplo, en lo referente a la disminución de los recursos hídricos y la regresión de la costa, la pérdida de la diversidad biológica y ecosistemas naturales, el aumento de los procesos de erosión del suelo y la pérdida de vidas y bienes derivadas de la intensificación de acaecimientos adversos asociados a fenómenos climáticos extremos, tales como inundaciones, incendios forestales y olas de calor (Peñuelas, Sabaté, Filella y Gracia, 2004; Costa, 2007; IPCC, 2007; CondeÁlvarez y Saldaña-Zorrilla, 2007), que afectan la dinámica socio ambiental de los pobladores.

Lo señalado anteriormente puede afectar los asentamientos humanos quienes son usuarios directos e indirectos de los recursos naturales. Asimismo, se convierten en actores particularmente importantes, debido a la rapidez con la que han ocurrido los cambios y a los daños que provocan sobre los bienes y servicios que se prestan en los litorales y a la forma en que los afecta (Altieri y Nicholls, 2008; Barton, 2009; Sepúlveda e Ibrahim, 2009). Actualmente, el número de habitantes y comunidades costeras está en aumento. En algunos casos, son centros económicos importantes donde se desarrollan actividades manufactureras, pesqueras, turísticas, petroleras y de prestación de servicios que desempeñan una función productiva, comercial y de servicios relevante (Aguilar y Castañeda, 2000).

En ese sentido, dichas actividades son el sustento económico de las familias que habitan en las zonas costeras. La creciente influencia de los cambios provocados por la variabilidad climática pone en riesgo la seguridad alimentaria de estas personas. Y la vulnerabilidad se acrecienta a medida que aumenta la densidad poblacional, se modifican los cuerpos de agua, se deforesta, se contamina, se sobrepasa la capacidad de carga del planeta y aumentan los gases de efecto invernadero, entre otros factores (Sharp, 2004; Conde-Álvarez y Saldaña-Zorrilla, 2007).

A pesar de tales presiones, las comunidades costeras se han enfrentado a las cambiantes condiciones que se han desarrollado desde hace varias décadas. El fenómeno se conoce 
como resiliencia al clima, un concepto que se ha desarrollado por la urgente necesidad de generar capacidades locales para enfrentar la variabilidad climática (Barton, 2009).

La resiliencia es concebida por diversos autores de distintas formas y niveles de profundidad. Estas visiones se han convertido en una limitación para el análisis holístico de los efectos e influencias de la variabilidad climática porque se proponen desde la perspectiva de los sistemas sociales hasta la de los sistemas biológicos o ecológicos. Es entendida como la mejora del sistema o de su capacidad para enfrentarse a los términos impulsores (Costa, 2007). Igualmente, se asocia con "la capacidad de un ecosistema para tolerar un grado de alteración sin colapsar determinándose un estado cualitativo diferente que, a su vez, resulta controlado por un conjunto diferente de procesos" (Resilience Alliance, 2002).

En tanto que Byg y Salick (2009) lo han propuesto como la capacidad de respuestas de comunidadesyactoreslocales, Walker,Carpenter, Anderies, Abel, Cumming, Janssen, Lebel, Norberg, Peterson y Pritchard (2002) proponen que la resiliencia es el potencial de un sistema de permanecer en una configuración particular y mantener las evaluaciones y funciones; consiste en la capacidad del sistema para volver a organizarse, luego de la perturbación impulsada por el cambio. Pero debe considerarse que, en ocasiones, se confunde con adaptación. En este documento se utiliza la definición propuesta por Walker et al. (2002).

Esta capacidad de organizarse y enfrentar las nuevas condiciones ha sido el motor que permite redireccionar parte de los esfuerzos globales, así como los fondos hacia la atención de la vulnerabilidad, incrementando la resiliencia y capacidadadaptativadelapoblaciónylossistemas productivos frente a la variabilidad climática.
Algunas herramientas que favorecen la resiliencia son el ordenamiento y la planificación territorial que contribuye a reducir los riesgos $y$, a la vez, promueve el bienestar ambiental y social. Dicho proceso minimiza la vulnerabilidad de las comunidades locales y permite identificar las oportunidades de mejora en las diversas prácticas (Lira, 2006). Pero requieren la integración de los componentes del sistema socioecológico para satisfacer las necesidades, generar capacidades de adaptación y establecer los límites del sistema (Gallopín, 2003).

Sin embargo, en términos generales, las instituciones de gestión ambiental, pesquera y agrícola tienen una limitada capacidad humana, financiera y técnica para ser eficaces en la implementación de medidas dirigidas a aumentar la resiliencia frente a la variabilidad climática, lo cual ocurre en otras áreas de la región centroamericana (African and Latino American Resilience to Climate Change Project ARCC, 2014). Además, las instituciones locales en la región han emprendido tímidas acciones que son la respuesta a la forma en que se identifican las vulnerabilidades.

En esta misma línea, IPCC (2013) asume que la vulnerabilidad frentealimpactodela variabilidad climática es una función de la exposición, la sensibilidad y la capacidad de adaptación de los sistemas. Por lo tanto, podemos entender que este fenómeno considerado antropocéntrico genera procesos resilientes en los sistemas socioecológicos, reconociendo la interacción e interdependencia de los seres humanos y la naturaleza, y de la forma en que los primeros dependen de los servicios ecosistémicos para lograr sus medios de vida.

A su vez, se ha generado dos corrientes de pensamiento en términos de resiliencia y adaptación. La primera es la adaptación basada en ecosistemas y la segunda, la basada en comunidades. Este último es el enfoque de 
análisis que se empleará en el documento actual.

La adaptación basada en comunidades es un proceso liderado por las comunidades humanas donde se consideran sus prioridades, necesidades y capacidades para que se empoderen para enfrentarlosimpactos dela variabilidad climática. Dicho enfoque se generó como consecuencia de que las poblaciones vulnerables a la pobreza, a las enfermedades, a la discriminación, etc., también son vulnerables a la influencia de la variabilidad climática (Huq y Reid, 2007).

Se ha desarrollado discusión sobre lo que se entiende por adaptación a la variabilidad climática y en algunos casos, se propone que es un asunto de infraestructura y/o técnico. No obstante, la creación de capacidades que permitan el uso y manejo de esa infraestructura y técnicas es fundamental para alcanzar el éxito (Conde-Álvarez y Saldaña-Zorrilla, 2007).

En este estudio se evalúan los mecanismos de adaptación a los impactos de la variabilidad climática sobre los ecosistemas marinos y costeros para la reducción de la vulnerabilidad de las comunidades costeras del Caribe Sur de Costa Rica.

\section{Metodología}

En esta sección se abordan la metodología empleada en la caracterización de la respuesta de las comunidades locales del Caribe Sur de Costa Rica ante la influencia de la variabilidad climática.

\section{Área de estudio}

Se encuentra dentro del Área de Conservación La Amistad Caribe (ACLAC) del Sistema Nacional de Áreas de Conservación (SINAC) del Ministerio de Ambiente y Energía (MINAE) que incluye los cantones de Talamanca y Limón, esta es una región policultural, rica en biodiversidad $\mathrm{y}$ tradiciones donde conviven aborígenes latinos $\mathrm{y}$ descendientes de chinos y afrocaribeños (ProAmbi, 1996).
Para fines de este estudio, el Caribe Sur de Costa Rica se delimitó desde el Cantón Central de la provincia de Limón hasta el río Sixaola, el cual forma una frontera natural con la República de Panamá (ICT, 2002) (figura 1).

La zona de vida corresponde a Bosque Húmedo Tropical (bhT) (Holdridge, 1967), es una región húmeda que alberga sistemas ecológicos que han permitido el desarrollo de actividades productivas y son importantes para la conservación delos recursosnaturales [Ministerio del Ambiente Energía y Telecomunicaciones (MINAET), Instituto Meteorológico Nacional (IMN), Programa de las Naciones Unidas para el Desarrollo (PNUD) y Comité Regional de Recursos Hidráulicos (CRRH), 2008] y forma parte de un pequeño corredor arrecifal que conecta las áreas de la costa Caribe de Nicaragua y de Panamá. Y en Costa Rica incluye los parches arrecifales del Parque Nacional Cahuita (PNC) y el Refugio Nacional de Vida Silvestre GandocaManzanillo (REGAMA).

El clima de la zona presenta una condición bimodal del periodo lluvioso; la primera se extiende de noviembre a marzo y la segunda de junio a agosto (Herrera, 1985), con precipitaciones de $4500 \mathrm{~mm} / \mathrm{año}$ (IMN, 2008). Las mareas son mixtas, principalmente de tipo diurnas y con un intervalo pequeño de 30 a $50 \mathrm{~cm}$. El oleaje entre enero y junio proviene del Noreste y del Este entre julio y diciembre, dependiendo de la posición de la Zona de Convergencia Inter Tropical. Las corrientes fluyen de Noroeste a Sureste con pequeños remolinos que viajan en dirección ciclónica, mediante las cuales se transportan sedimentos terrestres derivados de zonas ribereñas y de las áreas costeras deforestadas (Cortés y Risk, 1985).

Dentro de esta región denominada Caribe Sur se encuentran inmersas tres áreas protegidas de interés: el Refugio Nacional de Vida Silvestre Mixto Gandoca-Manzanillo, que fue creado en 


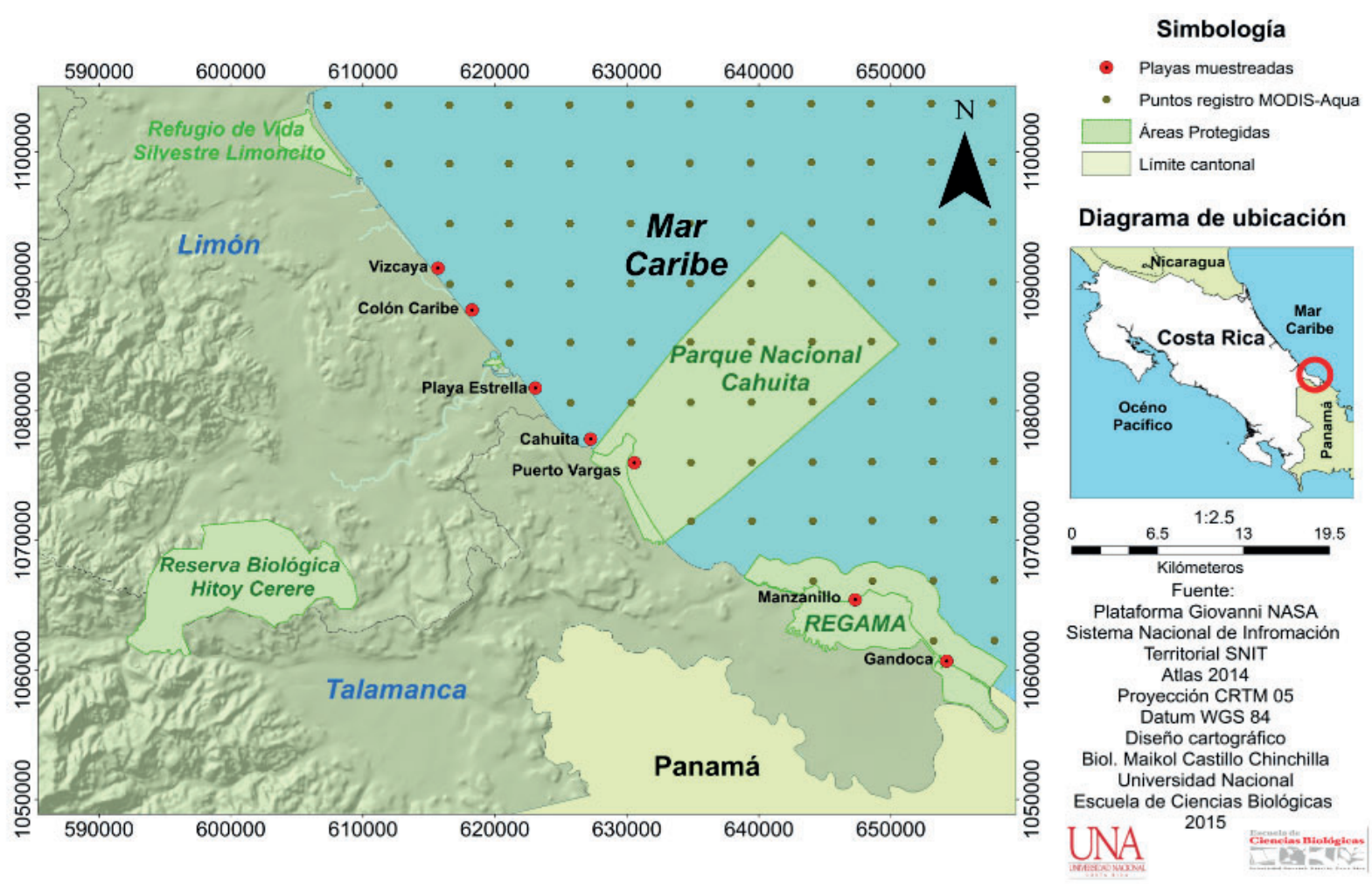

Figura 1. Área de estudio en el Caribe Sur, Limón, Costa Rica. Fuente: Piedra, Hernández, Sierra, Pereira, Castillo y Morales, 2018.

julio de 1985 mediante el Decreto Ejecutivo N. - $^{-}$ 16614-MAF01 y fue declarado sitio RAMSAR el 11 de diciembre de 1995; este posee una porción terrestre y otra marina (Weitzner y Fonseca, 2000).

El Parque Nacional Cahuita fue creado por el Decreto Ejecutivo N. 1236 -A, del 7 de septiembre de 1970, con el fin de proteger la flora y fauna, los arrecifes de coral y varios ecosistemas marinos (Quesada, 1997). De igual forma, presenta una porción terrestre y otra marina.

El Refugio Nacional de Vida Silvestre Limoncito (RNVS Limoncito) fue establecido por Decreto Ejecutivo N. 23121, en febrero de 1994, con declaración de refugio estatal. Al analizar la situación legal de los terrenos, se concluyó que la mayoría se encontraban inscritos a nombre de particulares. El decreto se modificó en mayo de 1994, en el artículo número uno y se cambia a la categoría de refugio con carácter mixto (Piedra-
Castro, Bravo y Salazar, 2009), que solamente tiene porción terrestre.

En las llanuras se desarrollan cultivos como banano, tubérculos y granos (Morales, 2000). Forma parte de la vertiente Caribe, con cuencas de gran importancia como Sixaola, Estrella, Banano y Bananito, entre otras. Durante la noche, predomina la brisa tierra-mar que viene del oeste y tiene baja velocidad. Es producto de un rotor acentuado a la costa, generando estabilidad en el viento. La precipitación anual es de 3000 a $2500 \mathrm{~mm}$.

\section{Materiales y métodos}

Se realizóla recopilación y sistematización de la información sobre los impactos de la variabilidad climática sobre los ecosistemas marinos y costeros a partir de los criterios de adaptación basada en comunidades. Para ello, se consultaron 
las bases de datos digitales de Academic Search Complete, Annual Reviews, Biological Abstracts, Current Contents, Environment y buscadores de internet. Se utilizaron palabras clave como ecosistemas marinos, ecosistemas costeros, aumento del nivel del mar, cambio climático, variabilidad climática, adaptación, mitigación con el operador booleano " $\mathrm{y}$ " o "and", tanto en español como en inglés, respectivamente.

Se utilizó la técnica de observación no participante y la encuesta según la metodología descrita por Aguilar y Castañeda (2000) para realizar un diagnóstico del uso de los recursos naturales en los ecosistemas marinos y costeros de interés, de los beneficios que reciben de estos espacios naturales, los impactos que perciben asociados a la variabilidad climática y los mecanismos que han desarrollado para enfrentar dichos cambios.

La observación no participante realizada de forma grupal se aplica cuando no se dispone de la confianza suficiente para participar en los eventos. Las observaciones y notas se realizaron como observador sin intervenir de la actividad analizada y se realizó durante reuniones comunales y en actividades diarias de los miembros de las comunidades (Creswell, 2005). Se aplicaron 65 encuestas para identificar las condiciones presentes en un espacio y un tiempo determinados. El instrumento consta de 15 preguntas relacionadas con las actividades socioeconómicas que desarrollan y las acciones que sellevan a cabo en relación con la variabilidad climática y su influencia en el área. El instrumento presentó una breve introducción, en la cual se explica el objeto de estudio y se indica la confidencialidad de las respuestas alinstrumento. Va seguido de un grupo de ítems sobre datos sociodemográficos como la edad, sexo, actividad económica que realiza, estudios, etc. seguidos de preguntas sobre el estado de los ecosistemas, del manejo, problemas comunales, etc.
De estas preguntas, 11 eran cerradas de marque con $\mathrm{X}$, la mayoría con dos opciones de respuesta; pero también había de opciones múltiples. Y se determinó la frecuencia de aparición de determinadas respuestas a partir de las categorías preestablecidas.

Se realizó la validación del contenido del cuestionario que se entendió como el grado en que su aplicación representa de forma adecuada lo que se propone valorar (Thomas y Nelson, 2007). Para alcanzar niveles óptimos de validez de contenido se utilizó la técnica de aplicación del instrumento fuera del área de estudio. Para conocer la validez del contenido desde la perspectiva de la comprensión de los sujetos objetos de estudio y la fiabilidad, se usó una matriz de cotejo que evaluó la pregunta, el motivo de confusión y el modo de respuesta.

La encuesta fue aplicada a 15 personas en la localidad de Piuta en Limón, seleccionadas al azar. Se valoró el grado en el que los entrevistados comprendían las preguntas del cuestionario, analizando los siguientes criterios: grado de comprensión del cuestionario mediante el registro de las preguntas, dudas y comentarios que hicieron sobre el instrumento durante la aplicación de este, así como los ítems que no fueron entendidos en la primera lectura. Posteriormente, se realizaron las adaptaciones necesarias al instrumento.

A partir de los datos obtenidos se realizó un inventario de los principales usos productivos que mantienen los pobladores y de los principales recursos naturales que son empleados para desarrollar tales actividades. Se confeccionó una taxonomía de usos de la zona costera en cuestión y se elaboraron las matrices de usos referidos a los recursos acorde con la metodología de Vallega (1999). Adicionalmente, se celebraron tres reuniones con actores claves en las localidades de Manzanillo, Cahuita, Puerto Viejo, Gandoca y Limón. 


\section{Resultados}

De las 65 encuestas aplicadas, fueron respondidas por mujeres $49 \%$ y $51 \%$ por hombres mayores de 18 años. El 32\% de los entrevistados eran proveedores únicos del núcleo familiar pero lo frecuente es que fuera combinado con hijos, cónyuge, compañeros sentimentales, primos o tíos. La mayoría de los entrevistados han concluido la secundaria (67\%). El 53\% han logrado una carrera técnica principalmente en el Instituto Nacional de Aprendizaje (INA) y el Colegio Universitario de Limón (CUN Limón).
Las principales actividades económicas de la zona costera fueron la agricultura de subsistencia con dominancia del cultivo de plátanos, banano, yuca y ñame. Aunque en el área, hay empresas transnacionales de cultivos intensivos de banano, estos no alcanzan la franja costera.

Se identificó que se desarrolla la pesca artesanal comercial con énfasis en cambute (Strombus gigas), langosta (Panulirus argus), róbalos, calva, pargos (Lutjanus sp), tiburones, macarela, kingfish y algunos camarones peneidos, por lo que es una pesquería artesanal multiespecífica (tabla 1). Destaca la actividad turística que puede contribuir de forma directa

Tabla 1

Principales especies de interés económico pesquero reportadas por los usuarios en el Caribe Sur

\begin{tabular}{lll}
\hline Familia & Especie & Nombre común \\
\hline Centropomidae & $*$ & Róbalos \\
Scorpaenidae ** & Pterois volitans/miles* & Pez león \\
Scombridae & Scomber scombrus & Tiburones \\
Scombridae & Scomberomorus cavalla & Macarela \\
Palinuridae & Panulirus argus & Kingfish \\
Lutjanidae & $*$ & Langosta espinosa \\
Centropomidae & $*$ & Pargos \\
Sphyraemidae & Sphyraena barracuda & Róbalo pequeño \\
Bothidae & Trichopsetta caribbea & Picuda \\
Sciaenidae & $*$ & Lenguado \\
Serranidae & Epinephelus striatus & Corvina \\
Serranidae & Epinephelus guttatus & Mero \\
Coryphaenidae & Coryphaena hippurus & Cabrilla \\
Istiophoridae & Kajikia albidus & Dorado \\
Scombridae & Thunnus sp. & Marlin blanco \\
Penaeidae & Farfantepenaeus notialis & Atún \\
Penaeidae & Xiphopenaeus kroyeri & Camarón rosado \\
Mugilidae & Mugil curema & Camarón tití \\
Belonidae & Ablennes hians & Lisa \\
\hline
\end{tabular}

*Se incluyen varias especies que con la información obtenida no se puede determinar a nivel de género y especie. Fuente: Piedra, Hernández, Sierra, Pereira, Castillo y Morales, 2016 
o indirecta a la economía familiar y en la que se puede emplear parte de los equipos que se emplean para la pesca.

Las principales artes de pesca empleados son buceo a apnea o pulmón, caña de pescar con señuelos y anzuelos. Estos se emplean para el aprovechamiento costero de las pesquerías como la langosta. Se realiza en las zonas someras empleando botes y motores o ingresando con equipo básico de buceo como aletas de buceo, careta y snorkel desde la costa. De igual forma, lanzan la cuerda y la caña desde la playa, las desembocaduras de los ríos y las áreas rocosas costeras, principalmente.

Para la pesca que ocurre alejada de la costa, sin que sea aguas profundas, se usan líneas palangreras, pequeñas redes de arrastre y nasas empleando botes o pangas elaborados con fibra de vidrio, de $8 \mathrm{~m}$ de eslora aproximadamente e impulsados por uno o dos motores fuera deborda.

La pesca costeraes desarrollada principalmente por hombres adultos y jóvenes, quienes alcanzan hasta $75 \%$ de la frecuencia. También participan niños de ambos géneros con 15\% de representación y 10\% corresponde a mujeres. En el caso de niños y las mujeres, se trata de una actividad recreativa $(88 \%)$, aporte de proteína por pesquería de subsistencia (12\%) y el resto como fuente de ingresos al grupo familiar. En el caso de los hombres adultos y jóvenes, la mayor parte de los usuarios $(89.6 \%$ ) realizan la actividad como fuente de ingresos al grupo familiar.

El destino final de la pesca puede ser el autoconsumo, como la fuente principal de proteína de la familia, la venta en la carretera que plantea problemas de control de la calidad sanitaria y comercializada en el mercado local y nacional mediante venta directa o intermediarios (figura 2). Los recibidores de pescado o centros de acopio son escasos en la zona.

La actividad turística se aborda desde diversos frentes. Algunas personas se dedican al transporte marítimo para trasladar turistas a sitios de pesca deportiva, a arrecifes coralinos y a playas, como principales destinos. Otros incluyen el trabajo como guías turísticos de buceo apnea o pulmón y autónomo. Algunos de

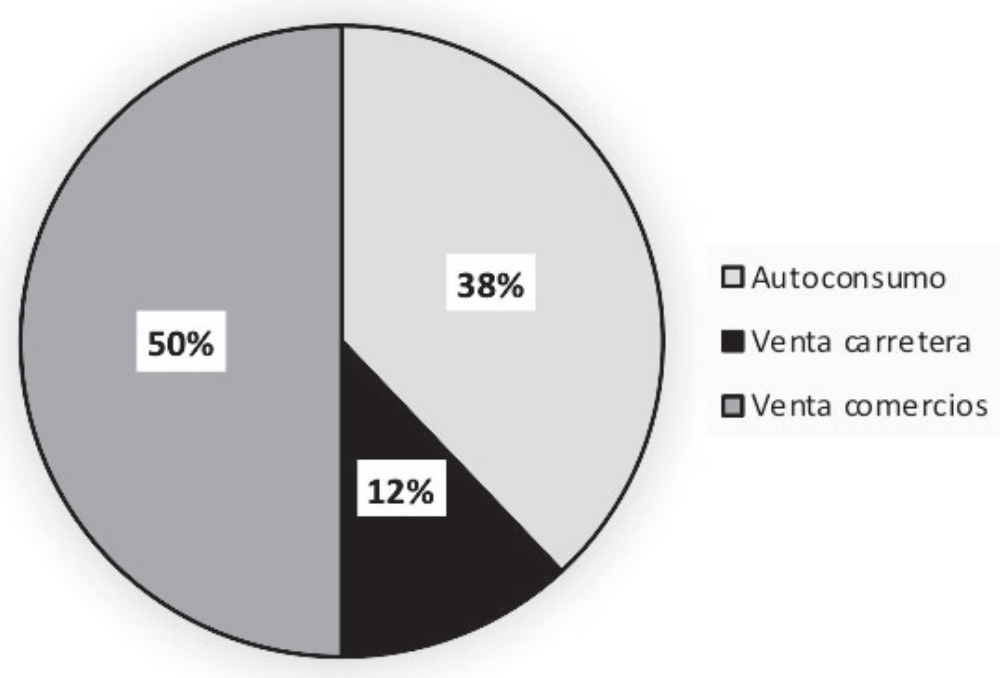

Figura 2. Destino final del producto pesquero en el Caribe Sur de Costa Rica. Fuente: Piedra, Hernández, Sierra, Pereira Chávez, Castillo y Morales, 2018 
los guías cuentan con el curso del INA en tanto que otros son empíricos.

Otros pobladores realizan recorridos guiados por senderos terrestres principalmente en el PNC y el REGAMA. Algunos son guías locales certificados, principalmente en el PNC, pero la mayoría no cuenta con esto. A lo anterior se suma la industria del alojamiento a baja escala y la gastronómica. El máximo desarrollo local de estas actividades ocurre en las localidades de Puerto Viejo, Cahuita y Manzanillo.

De menor importancia se encuentra el comercio con pequeños supermercados, bazares, tiendas de recuerdos o souvenires, ropa y electrodomésticos. Los comercios grandes y surtidos se ubican en Puerto Viejo y en la ciudad de Limón.

La recolección de cocos para producir el aceite respectivo y comercializarlo es también una actividad importante. Los habitantes han cultivado esta palma en la zona marítima terrestre donde no se encuentran limitados para recolectar el fruto. La recolección está asociada a la extracción del aceite de coco crudo o cocido que es empacado en envases plásticos reutilizados. Esta es una actividad económica informal que provee ingresos económicos a los miembros de las comunidades locales, de forma ocasional, producto de la venta en las márgenes de la calle o a intermediarios que vienen desde San José u otras localidades.

Los usos que las comunidades y los visitantes realizan de los ecosistemas analizados permiten diferenciar entre el uso de los recursos vivos y los inertes que pueden ser de carácter extractivo o no extractivo. Se destacó la agricultura comercial familiar y comercial intensiva (52\%), el uso de recursos vivos extractivos de la pesca de diversas especies en las modalidades planteadas (29\%), el comercio (10\%) y subsistencia (8\%). También, incluyeron el cultivo del cacao, con un enfoque orgánico (1\%) que tiene un apogeo reciente.

En cuanto al uso no extractivo de recursos naturales, la actividad más importante que se realiza es el turismo tanto en la parte marina como en la terrestre. La flora y fauna continental y marina son recursos cada vez más empleados que aportan a la economía local (tabla 2).

Por otra parte, a partir de los grupos focales se identificó que los poblados analizados, excepto la zona urbana de la ciudad de Puerto Limón, se caracterizan por ser asentamientos de tipología rural, próximos a la línea de costa o limitando con la playa. No presentan sistemas de alcantarillado sanitario, por lo que los desechos líquidos domésticos y el escurrimiento terrestre llegan sin tratamiento al mar.

Otra característica de la zona es la presencia de infraestructura ubicada en la zona marítima terrestre, la cual está limitada en la ley de este mismo nombre, con número 4063. Entre las infraestructuras que se pueden observar están la carretera de la ruta 36 que se mantiene paralela a la costa. Asimismo, se identifican viviendas instaladas en esta zona (figura 3).

Por ejemplo, la caracterización de la franja costera entre el río Vizcaya y el Estero Negro mostró que este sector posee una longitud de $7.5 \mathrm{~km}$ línea de costa con un total de 25 casas de habitación dentro de la Zona Marítima Terrestre. Se pudo observar unidades habitacionales tanto en la zona pública como en la restringida.

Las comunidades locales identifican una serie de cambios en los ambientes marinos y costeros que impactan las actividades que realizan, así como la calidad de vida. Tales modificaciones las asocian con efectos e influencias provocados por la variabilidad climática. Se identificaron cuatro efectos percibidos por los locales.

El primero fue el aumento sobre el nivel del mar, al que le asociaron nueve acciones de 
Tabla 2

Principales usos por localidad en el Caribe Sur de Costa Rica, 2016

\begin{tabular}{|c|c|c|c|}
\hline Localidad & Usos & Ecosistema & Comunidad \\
\hline Isla Uvita & $\begin{array}{l}\text {-Buceo a pulmón } \\
\text {-Buceo autónomo } \\
\text {-Pesca para acuarios } \\
\text {-Pesca autoconsumo } \\
\text {-Transporte acuático }\end{array}$ & Arrecifes coralinos & $\begin{array}{l}\text { Limonenses } \\
\text { Turistas nacionales } \\
\text { Turistas extranjeros }\end{array}$ \\
\hline Cahuita & $\begin{array}{l}\text {-Buceo a pulmón } \\
\text {-Buceo autónomo } \\
\text {-Pesca autoconsumo } \\
\text {-Pesca de langostas } \\
\text {-Turismo } \\
\text {-Preservación } \\
\text {-Aceite de coco } \\
\text {-Transporte acuático }\end{array}$ & $\begin{array}{l}\text { Arrecife coralinos } \\
\text { Pastos } \\
\text { Playa } \\
\text { Manglar }\end{array}$ & $\begin{array}{l}\text { Limonenses } \\
\text { Turistas nacionales } \\
\text { Turistas extranjeros } \\
\text { SINAC }\end{array}$ \\
\hline Puerto Viejo & $\begin{array}{l}\text {-Buceo a pulmón } \\
\text {-Buceo autónomo } \\
\text {-Pesca para acuarios } \\
\text {-Pesca autoconsumo } \\
\text {-Turismo } \\
\text {-Comercio } \\
\text {-Transporte acuático }\end{array}$ & $\begin{array}{l}\text { Arrecife } \\
\text { Pastos } \\
\text { Playa }\end{array}$ & $\begin{array}{l}\text { Limonenses } \\
\text { Turistas nacionales } \\
\text { Turistas extranjeros }\end{array}$ \\
\hline Manzanillo & $\begin{array}{l}\text {-Buceo a pulmón } \\
\text {-Buceo autónomo } \\
\text {-Pesca para acuarios } \\
\text {-Pesca autoconsumo } \\
\text {-Turismo } \\
\text {-Comercio } \\
\text {-Conservación } \\
\text {-Transporte acuático } \\
\text {-Aceite de coco }\end{array}$ & $\begin{array}{l}\text { Arrecife } \\
\text { Pastos } \\
\text { Playa }\end{array}$ & $\begin{array}{l}\text { Limonenses } \\
\text { Turistas nacionales } \\
\text { Turistas extranjeros } \\
\text { SINAC }\end{array}$ \\
\hline Gandoca & $\begin{array}{l}\text {-Buceo a pulmón } \\
\text {-Buceo autónomo } \\
\text {-Pesca autoconsumo } \\
\text {-Turismo } \\
\text {-Voluntariado } \\
\text {-Turismo } \\
\text {-Transporte } \\
\text {-Pesca subsistencia }\end{array}$ & $\begin{array}{l}\text { Arrecife } \\
\text { Playa } \\
\text { Manglar }\end{array}$ & $\begin{array}{l}\text { Limonenses } \\
\text { Turistas nacionales } \\
\text { Turistas extranjeros } \\
\text { Voluntarios }\end{array}$ \\
\hline
\end{tabular}

Fuente: Piedra, Hernández, Sierra, Pereira Chávez, Castillo y Morales, 2018 
Caracterización de la respuesta de las comunidades locales a la influencia directa de la variabilidad climática

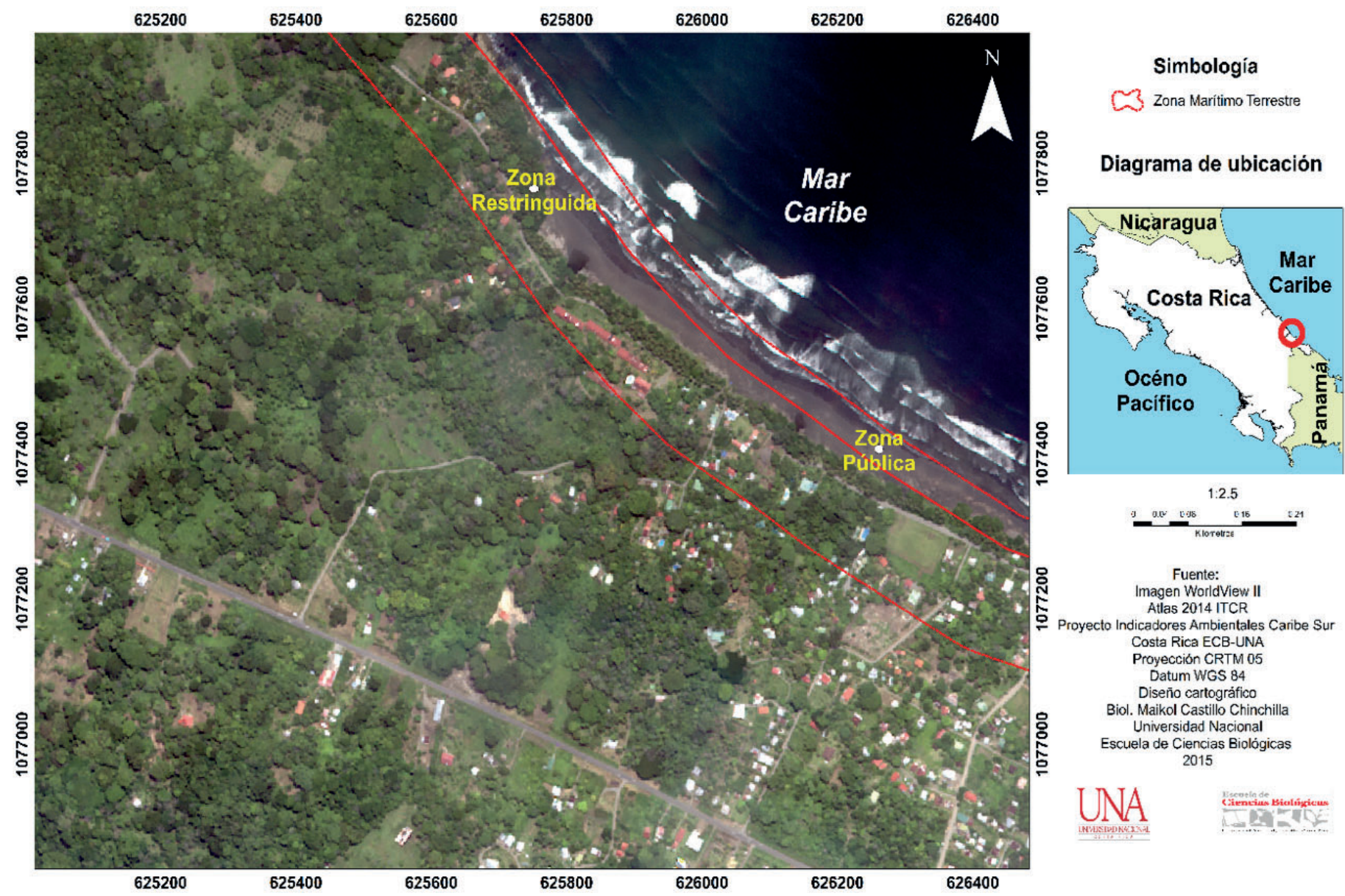

Figura 3. Ubicación de infraestructura en la zona pública y restringida de la zona marítima terrestre. Fuente: Piedra, Hernández, Sierra, Pereira, Castillo y Morales, 2018 
impacto y que se agruparon en nueve grupos de impactos. El segundo efecto fue el aumento de la temperatura atmosférica con 12 acciones y agrupadas en 11 impactos. El tercer fue el aumento de la temperatura superficial del mar donde propusieron 4 acciones que se pueden agrupar en 6 impactos posibles. En tanto que el último fue aumento del $\mathrm{CO}$, al que se le asoció una única acción y un único impacto (tabla 3).
Ante los inminentes cambios observados por las comunidades locales les ha impulsado a proponer y desarrollar soluciones que les permitan mantenerse viviendo en la zona y sustentar sus necesidades básicas. Estas respuestas contribuyen a reducir los desplazados por la variabilidad climática por la capacidad de resiliencia que tienen los locales. Dichas respuestas locales atendieron seis amenazas mediante 16 soluciones (tabla 4).

\section{Tabla 3}

Principales impactos percibidos por las comunidades locales con relación a la variabilidad climática, Caribe Sur, Costa Rica, 2016.

\begin{tabular}{|c|c|c|}
\hline Efecto & Acción & Impacto \\
\hline \multirow{9}{*}{$\begin{array}{l}\text { Aumento del } \\
\text { nivel del mar }\end{array}$} & Rompe la tubería & Suministro de agua potable \\
\hline & Daños a caminos & Libre tránsito \\
\hline & Daños a casas & Vivienda digna \\
\hline & Intrusión de agua salada & Aguas subterráneas \\
\hline & Pérdida cultivos de subsistencia & Seguridad alimentaria \\
\hline & Playas de menor calidad & Turismo y recreación \\
\hline & Abandono de viviendas & Desplazamiento de comunidades \\
\hline & $\begin{array}{l}\text { Invertir en reparar calles y caminos ubicados en } \\
\text { la cercanía de la costa }\end{array}$ & Comunicación terrestre \\
\hline & $\begin{array}{l}\text { Cambio en la vegetación de los pantanos } \\
\text { cercanos a la costa }\end{array}$ & Biodiversidad \\
\hline \multirow{6}{*}{$\begin{array}{l}\text { Aumento } \\
\text { de la } \\
\text { temperatura } \\
\text { atmosférica }\end{array}$} & Uso de ventiladores & Consumo energía \\
\hline & Reducción de la abundancia de peces & Seguridad alimentaria \\
\hline & Regar jardines y cultivos & Consumo de agua \\
\hline & Casas más calientes & Bienestar humano \\
\hline & Aumento de las poblaciones de zancudos & $\begin{array}{l}\text { Riesgo de enfermedades por } \\
\text { vectores }\end{array}$ \\
\hline & Inundaciones por agua marina & $\begin{array}{l}\text { Pérdida de infraestructura y de } \\
\text { fincas }\end{array}$ \\
\hline
\end{tabular}




\begin{tabular}{|c|c|c|}
\hline \multirow[t]{6}{*}{$\begin{array}{l}\text { Aumento } \\
\text { de la } \\
\text { temperatura } \\
\text { atmosférica }\end{array}$} & $\begin{array}{l}\text { Aumento de tormentas, vientos fuertes y hasta } \\
\text { huracanes }\end{array}$ & $\begin{array}{l}\text { Pérdida de infraestructura y } \\
\text { vegetación } \\
\text { Riesgo a la vida humana }\end{array}$ \\
\hline & Aparición de especies introducidas & Seguridad alimentaria \\
\hline & Cambios en la productividad agrícola & Seguridad alimentaria \\
\hline & $\begin{array}{l}\text { Aumento de enfermedades por el esfuerzo } \\
\text { realizado }\end{array}$ & Salud pública \\
\hline & Necesidad de emplear bloqueador solar & Salud pública \\
\hline & $\begin{array}{l}\text { Más zancudos con peligro de ser portadores de } \\
\text { enfermedades como dengue, paludismo, fiebre } \\
\text { amarilla }\end{array}$ & Salud pública \\
\hline \multirow{4}{*}{$\begin{array}{l}\text { Aumento } \\
\text { de la } \\
\text { temperatura } \\
\text { superficial } \\
\text { del mar }\end{array}$} & $\begin{array}{l}\text { Aumento de tormentas, vientos fuertes y hasta } \\
\text { huracanes }\end{array}$ & $\begin{array}{l}\text { Pérdida de infraestructura y } \\
\text { vegetación } \\
\text { Riesgo a la vida humana }\end{array}$ \\
\hline & Reducción de la abundancia de peces & Seguridad alimentaria \\
\hline & Cambio en las especies de peces presentes & Impacto en la biodiversidad \\
\hline & Más biofouling adherido a las lanchas & Reducción vida útil de los equipos \\
\hline $\begin{array}{l}\text { Aumento } \\
\text { del CO2 }\end{array}$ & Reduce la producción de alimentos & Seguridad alimentaria \\
\hline
\end{tabular}

Fuente: Piedra, Hernández, Sierra, Pereira, Castillo y Morales, 2018. Datos obtenidos a partir de reuniones con actores claves.

\section{Tabla 4}

Soluciones propuestas para mitigar los posibles impactos de la variabilidad climática sobre las comunidades locales, Caribe Sur, Costa Rica, 2016

Amenazas Soluciones

Cambio en la precipitación, temperatura y corrientes marinas

Erosión costera
-Pesca suplementaria a actividades económicas -Agricultura orgánica: cacao, yuca, ñame y guanábana -Agricultura extensiva: banano, plátano y yuca

-Voluntariado diversificado: trabajo con escuelas y colegios, en las fincas, en actividades de patrullaje, etc. 
Pérdida infraestructura costera

Reducción de voluntarios para la observación de tortugas
-Traslado de infraestructura a zonas alejadas de la costa -Reforestación de la línea costera para proteger calles, caminos y carreteras

-Protección a pantanos y bosques inundados de yolillo -Reubicación de pobladores costeros por el riesgo asociado y la disminución de oportunidades laborales

-Voluntarios que participan en actividades complementarias como educación ambiental en escuela y colegios, trabajo en fincas orgánicas y patrullaje en áreas protegidas, etc. -El desarrollo de programas de gestión local con la participación de los grupos locales -incorporación de costumbres y tradiciones en la oferta turística

-Desarrollo un protocolo para atender las amenazas del pez león.

-Desarrollo de programas educativos y de sensibilización local para informar sobre la amenaza del pez león y su impacto sobre la biodiversidad -Desarrollo de artes de pesca locales (nasas y arpones) -Comercialización de peces

-Algunas fincas están adscritas al pago de servicios ambientales por protección de bosques

Fijación de carbono

Fuente: Elaboración propia con datos obtenidos en reuniones con actores claves.

\section{Discusión}

En algunas de las comunidades locales, donde la pesca ha sido una actividad alternativa o suplementaria de una actividad económica principal, han identificado una disminución en el acceso al recurso pesquero. Entonces, se han abocado a la introducción de otras actividades productivas como la agricultura orgánica, que les permite obtener un producto comercializable con valor agregado y el acceso a turistas y voluntarios.
Según los datos obtenidos de los pobladores, estos manifestaron que se presenta una disminución de la calidad de las playas de anidación de tortugas. Esta afecta la observación por parte de este grupo que, durante años como producto de la presencia de tales especies, recibió importante visitación turística y actividades de voluntariado, lo que les impulsó a generar la ampliación de la oferta turística.

La ampliación de la oferta turística ha permitido beneficiar a las comunidades locales 
con la ejecución de proyectos de educación ambiental en las escuelas y los colegios, al igual que mejorar la infraestructura comunitaria como EBAIS, salones comunales y caminos. Asimismo, ha habido la incorporación del trabajo de los voluntarios en las fincas orgánicas que le ofrece una experiencia diferente, la participación en patrullaje de otros recursos diferentes a las tortugas en las áreas protegidas, la incorporación de costumbres y tradiciones que fomenten el interés del visitante, que a la vez les ha permitido a los locales consolidar micro y medianas empresas.

Desdeel2009, se reportóla invasión del pezleón en el área de estudio. Esta especie se considera una amenaza porque impacta negativamente las poblaciones de peces como depredadores topes y sobre los arrecifes de coral. El daño se produce inicialmente al nivel ecológico y posteriormente, a nivel económico debido a que limita la oferta turística y la producción pesquera. Para revertir estos procesos, las organizaciones pesqueras locales han desarrollado proyectos para pescarlo en nasas, han desarrollado protocolos para el manejo del pez león (Espinoza y Grandas, 2015), campañas educativas, torneos de pesca, entre otras medidas.

En este marco de acción, Beita y Sandoval (2011) proponen que la dinámica económica de Cahuita se fundamente en un mosaico de usos, que cambian constantemente con apoyo en la disponibilidad y las presiones que se reciban del entorno. Por tanto, las medidas de adaptación basada en las comunidades son una dinámica social de la región que ha permitido que los pobladores se mantengan en el largo plazo.

La población reconoce los beneficios aportados por este modelo económico sustentado en diferentes fuentes de trabajo, ya que logran el mejoramiento del bienestar humano de los habitantes que les permite lograr ingresos para suplir necesidades básicas, el acceso a proteína de diversos orígenes; asimismo, tienen la oportunidad de participar en actividades de educación ambiental y el apoyo a los servicios básicos, el contacto con personas de diversas latitudes y la posibilidad de aprender un segundo idioma. Tales condiciones fueron similares a las reportadas por Beita y Sandoval (2011).

Estos efectos asociados con la variabilidad climática pueden acentuarse cuando la percepción de los visitantes (turistas o voluntarios) se asocia con afectación de los ingresos por concepto de actividad turística y de la imagen conservacionista del país.

Las comunidades del Caribe Sur han sufrido cambios en los sistemas naturales en los que desarrollan sus actividades. Por ejemplo, el mar ha ingresado a sus fincas, se han inundado sus patios, se han salinizado los pantanos o han cambiado las especies vegetales por exposición al agua salada, etc. Estos cambios han provocado que los pobladores realicen acciones de manejo a partir de sus experiencias de vida.

Adicionalmente, los participantes perciben con menor frecuencia, el apoyo de las instituciones gubernamentales y no gubernamentales. Este sentir es destacado por Mora (2014, p. 1), quien menciona que "las comunidades conocen mejor las vulnerabilidades y riesgos presentes a nivel local, por lo que podrían gestionar con mejores resultados su territorio, descentralizando las acciones marino costeras frente al fenómeno climático".

Por consiguiente, las políticas de adaptación se hacen necesarias debido a los pronósticos de incrementos de temperatura entre 1.5 y $4.5^{\circ} \mathrm{C}$ para el año 2100 (IPCC, 2013). Las experiencias, las políticas y la investigación sobre adaptación a la variabilidad climática se han acrecentado en los últimos años (Dirección de Cambio Climático Ministerio de Ambiente y Desarrollo Sostenible de la Republica de Colombia, 2013). Algunas de 
las líneas estratégicas que se han impulsado son la adaptación basada en tecnologías e infraestructura, la adaptación basada en ecosistemas y la adaptación basada en comunidades. Estas herramientas han contribuido a enfrentar los efectos e influencias de la variabilidad climática y reducir la vulnerabilidad de las comunidades humanas.

Algunasdeestasestrategiassehandesarrollado de forma espontánea por las comunidades y en la actualidad, permiten facilitar la comunicación y el intercambio de información entre actores. La adaptación basada en comunidades muestra un enfoque reciente en cuanto a sus conceptos y metodologías (Dirección de Cambio Climático, Ministerio de Ambiente y Desarrollo Sostenible de la Republica de Colombia, 2013).

Las comunidades locales han mostrado vasta capacidad para resolver los problemas incipientes con relación a la influencia de la variabilidad climática. De todas maneras, las instituciones gubernamentales y no gubernamentales deben aportar a estos procesos para planificar y facilitar la adaptación en el mediano y largo plazo y con una visión holística de la relación ecosistema-comunidades que incorpore los tres ejes del desarrollo sostenible: ambiental, social y económico.

Sin embargo, Aragón, Colque y Rosales, (2012) señalan que son insuficientes los programas y proyectos enfocados a estrategias locales de adaptación a la variabilidad climática lo que incide en una limitada acción regional.

Aunque existen diversos casos en el mundo que analizan comunidades desplazadas como producto de la variabilidad climática (Castles, 2003; Brown, 2008; Gemenne y Reuchlin, 2008), (Romer, 2006), las comunidades analizadas no han alcanzado tal condición. Probablemente, porque han logrado resolver desde sus experiencias de vida las adversidades a las que se afrontan debido a que han mostrado resiliencia a estas condiciones. No obstante, si los cambios continúan ocurriendo y aumentando su intensidad, estas poblaciones podrían convertirse en desplazados ambientales en el futuro.

Por ende, es fundamental que se genere investigación sobre las tendencias de estos impactos. De ese modo, si fuese necesaria la migración, que sea planificada y no forzada (Jiménez y Suescún, 2011). Este tipo de migraciones ofrece la posibilidad de planificar, de considerar los impactos de la zona, de organizar con tiempo suficiente el traslado de forma tal que se reduzca la vulnerabilidad de las personas (Herrera 2006).

Esta planificación debe concretarse entendiendo que la variabilidad climática es un fenómeno complejo y que se comporta de diversas formas dependiendo de la latitud donde se analice. Por eso, no es posible generar un modelo o estrategia única, sino que debe partirse de la planificación desde lo local siendo que considere aspectos sociales, culturales, económicos y que dependerá de las características de la zona, de la sociedad y de la importancia medioambiental según lo plantea el Informe sobre Cambio Medioambiental y Migración Forzada (Jiménez y Suescún 2011).

A pesar de que el $\operatorname{IPCC}(2007,2013)$ plantea que está ocurriendo la acidificación del mar, los locales del área de estudio no reportan ningún cambio asociado a ese impacto. Tal vez porque los sistemas productivos locales no dependen de los moluscos siendo imperceptible tales cambios para ellos. Sin embargo, hay evidencia experimental (Orr et al, 2005) de que los corales y el plancton podrían presentar problemas para mantener sus esqueletos externos que están constituidos principalmente de carbonato de calcio, lo que afectaría la cadena trófica $\mathrm{y}$, por ende, la seguridad alimentaria de las comunidades locales que emplean la proteína de los organismos marinos para suplir las necesidades básicas. 
De igual forma, Hoegh-Guldberg y colaboradores (2007) plantean que las comunidades de arrecifes serán menos diversas y las estructuras arrecifales contendrán menos carbonato por la dificultad de incorporarlo. El fenómenodelasimplificación delascomunidades coralinas ha sido reportado en Cuba por Alcolado y otros (2010), este impacta la calidad de los ecosistemas, pero también las economías locales costeras que han apostado a la actividad turística de buceo con snorkeling y autónomo. Este impacto podría incidir en la economía local a futuro. En ese sentido, es fundamental el incremento de la colaboración entre el Sistema Nacional de Áreas de Conservación, específicamente el Área de Conservación La Amistad Caribe, las universidades, los colegios universitarios, el Instituto Costarricense de Turismo (ICT) y el Instituto Mixto de Ayuda Social (IMAS), entre otros actores sociales, al igual que las comunidades rurales y urbanas de diversos orígenes y costumbres que habitan en la zona y las organizaciones no gubernamentales que trabajan en la zona.

Los principales esfuerzos que deben realizarse están concentrados en la capacitación sobre el tema de la variabilidad climática, su influencia sobre las comunidades humanas y de los ecosistemas, la manera de reducir desde nuestras casas y actividades económicas las emisiones de gases con efecto invernadero, etc.

Otro aspecto relevante es el fortalecimiento en términos del cumplimiento de las normas legales vigentes como de la Ley de la Zona Marítima Terrestre (ZMT), el mantenimiento de las zonas de protección de cuerpos de agua donde se incluyen ríos y nacientes; las normas relacionadas con el uso y aplicación y agroquímicos, entre otros.

Tres aspectos fundamentales son: el análisis y aplicación de los caudales ecológicos de forma dinámica que impulse actividades agrícolas amigables con el ambiente; las políticas en uso racional del agua en todos los sectores como el agrícola, industrial y doméstico; y la temática de manejo adecuado de desechos líquidos, sólidos y gaseosos para reducir los aportes de estos al cambio global.

Debe asumirse el tema de gestión del riesgo en ecosistemas marinos y costeros como una prioridad que incluya la capacitación, el empoderamientolocal, la búsqueda de soluciones desde las experiencias de las comunidades locales, incorporando la variabilidad climática como una variable de gestión de los recursos que promueva los procesos de adaptación tanto de ecosistemas como de comunidades humanas.

En ese sentido, las áreas costeras son susceptibles a las acciones desarrolladas en el continente porque se ubican en las zonas bajas de las fuentes de contaminación urbanas, industriales y agrícolas. Además, las áreas con concentración urbana o semi-urbana ubicados en la costa o cerca de ella y la presión ambiental que ocasionan reduce la capacidad de carga de los ecosistemas que absorben los impactos (Roberts y Muir, 1995).

En el eje de investigación, debe priorizarse la actualización del inventario nacional de humedales en esta área de conservación, mejorar nuestro conocimiento sobre los cambios que ocurren en los sistemas naturales en relación con las variabilidades climáticas actuales, el estudio de las tenencias de la tierra en áreas de humedales, el fomento de la conciencia pública sobre estos temas y la reducción de la deforestación en el área.

Deben realizarse trabajos en restauración de los humedales, principalmente de los bosques de yolillo (Raphia taedigera), los cuales han cambiado de forma importante en la región (Castillo, 2014). Y, de forma conjunta, reducir el cambio de uso del suelo en humedales. 
También, debe fortalecerle y retomarse el tema de reconocimiento de los servicios ambientales de los humedales (PSA), así como la búsqueda de fuentes de energía alternas que sustituyan las represas por la variabilidad en los patrones de precipitación que se muestran en la zona.

A nivel institucional y local, deben promoverse programas de manejo de desechos sólidos y líquidosqueconsiderenlaseparación, recolección y tratamiento de los mismos, los cuales en la región están limitados. De igual forma, se deben fomentar de los planes institucionales de uso de la energía y del agua, en el diseño de infraestructuras nuevas debe considerarse el riesgo de afectación por los cambios en la línea de costa, la erosión costera y la fuerza del mar.

Lo anterior es fundamental para asegurar que las obras se mantengan en el largo plazo y se reduzca la necesidad de intervención posterior, así como la posibilidad de implementar sistemas de recolección de agua de lluvia.

Estas acciones son primordiales, ya que las leyes, las políticas y las estrategias no son indicadores confiables del nivel de compromiso que como país tenemos para enfrentar la variabilidad climática y sus efectos. En ese sentido, Aguilar (2015) señala que no se evidencia una correlación positiva entre la eficacia de la respuesta política y la implementación de medidas de respuesta. Por tal razón, la adaptación basada en comunidades corresponde un indicador de los avances en mitigación.

En la zona, se deben impulsar esfuerzos para que la acuicultura se desarrolle bajo criterios de sostenibilidad y reduciendo los impactos ambientales, dado que se ha demostrado que puede contribuir con la seguridad alimentaria que satisfaga la demanda local y nacional. Es indispensable debido a que las pesquerías de especies comerciales han reducido el volumen cosechado (GESAMP, 2001). Pero los procesos de producción y funcionamientos de la acuicultura deben administrarse de forma multidisciplinaria para mantener el delicado y complejo equilibrio de los ecosistemas costeros (Macintosh y Phillips 1993; Landesman 1994; Hopkins 1996).

Cada ciudadano debe asumir las responsabilidades asociadas con los cambios globales a los que nos enfrentamos. $Y$, adicionalmente, contribuir desde los diversos frentes que asume, desde el conocimiento y actividad económica que realiza hasta en el nivel individual como en el colectivo en el que participa. Asimismo, el desarrollo socio-económico, el ambiente y la variabilidad climática se entrelazan provocando impactos que no pueden ser obviados, sino que deben prevenirse. Se concluye que las comunidades costeras del Caribe Sur se han adaptado a las diversas condiciones que la variabilidad climática les ha presentado. Sin embargo, esto no es planificado.

\section{Referencias}

African and Latino American Resilience to Climate Change Project (ARCC). (2014). Vulnerabilidad y resiliencia frente al cambio climático en el Occidente de Honduras. Recuperado de https://www.agrilinks.org/sites/default/files/resource/files/Occidente $\% 20 \mathrm{de} \% 20$ Honduras $\% 20$ evaluacion $\% 20$ climatica_espanol_al\%20final.pdf

Aguilar, L. y Castañeda. I. (2000). Sobre marinos, marinas, mares y mareas: perspectivas de género en zonas marino - costeras. San José, Costa Rica: Unión Mundial para la Naturaleza, UICN.

Aguilar, Y. (2015). Legislación sobre Cambio Climático: un desafío para ElSalvador. Perspectivas, 1-14.

Alcolado, P. M., Caballero-Aragón, H., Lara, A., Rey-Villiers, N., Arriaza, L., Lugioyo, G., Alcolado-Prieto, P., Castellanos, S., Perera, S. y Rodríguez-García, A. (2013). Resiliencia en crestas de arrecifes coralinos del este del golfo de Batabanó, Cuba, y factores determinantes probables. Serie Oceanólogica, 13, 49-75. 
Altieri, M. A. y Nicholls, C. (2008). Los impactos del cambio climático sobre las comunidades campesinas y de agricultores tradicionales y sus respuestas adaptativas. Agroecología, 3, 7-24 pp.

Anadón, R., Duarte, C. M. y Fariña, C. (2005). Impactos sobre los ecosistemas marinos y el sector pesquero. En Moreno, J. M., De la Rosa, D. y Zazo, C. (Ed). Evaluación preliminar de los impactos en España por efecto del cambio climático (147-182). Castilla-La Mancha, España.

Aragón, O., Colque, P. y Rosales, B. (2012). Estrategia Local de adaptación al cambio climático en zonas marinas y costeras del Caribe Sur de Costa Rica. San José, Costa Rica: CATIE.

Barton, J. R. (2009). Adaptación al cambio climático en la planificación de ciudades-regiones. Revista de geografía Norte Grande, 43, 5-30.

Brown, O. (2008) Migración y cambio climático. Serie de EstudiosdelaOIMsobrelaMigración. Ginebra,Suiza: Organización Internacional para las Migraciones.

Byg, A. y Salick, J. (2009). Local perspectives on a global phenomenon- Climate change in Eastern Tibetan villages. Global Environmental Change, 19(2), 156-166.

Carballo, J. L., Bautista-Guerrero, E., Nava, H. y Cruz-Barraza, J. A. (2010). Cambio climático y ecosistemas costeros. Bases fundamentales para la conservación de los arrecifes de coral del Pacífico Este. La Biodiversidad en Ecosistemas Marinos y Costeros del Litoral de Iberoamérica y el Cambio Climático: I. Red Cyted Biodivmar, La Habana.

Castillo, M. (2014). Análisis espacial y temporal de los humedales costeros del Caribe Sur de Costa Rica entre 1989-2014. (Tesis de maestría). Universidad Nacional, Costa Rica.

Castles, S. (2003). La política internacional de la migración forzada. Migración y Desarrollo, 1, 7490.

Conde-Álvarez, C. y Saldaña-Zorrilla, S. (2007). Cambio climático en América Latina y el Cari- be: Impactos, vulnerabilidad y adaptación. $R e-$ vista Ambiente y Desarrollo, 23(2), 23-30.

Cortés, J., M. y Risk. J. (1985). A reef under siltation stress: Cahuita, Costa Rica. Bulletin of Marine Science, 36, 339-356.

Costa, C. (2007). La adaptación al cambio climático en Colombia. Revista de Ingeniería, 26, 74-80.

Creswell, J. W. (2005). Educational research: Planning, conducting and evaluating quantitative and qualitative research. Upper Saddle River USA: Pearson Education

Dirección de Cambio Climático Ministerio de Ambiente y Desarrollo Sostenible de la República de Colombia. (2013). Adaptación basada en Comunidades - AbC. Bases conceptuales y guía metodológica para iniciativas rápidas de $\mathrm{AbC}$ en Colombia. Recuperado de https://www.minambiente.gov.co/ images/Atencion_y_particpacion_al_ciudadano/ Consulta_Publica/030214_consulta_pub_adaptacion_comunidades.pdf

Espinoza, C. y Grandas, L. (2015). Protocolo para la captura, extracción y disposición final del pez león (complejo Pterois volitans/miles) en el Caribe Costarricense. Fundación Trichecus y Asociación de pescadores Artesanales del Caribe Sur.

Felicísimo, A. M., Muñoz, J., Mateo, R. G. y Villalba, C. J. (2012). Vulnerabilidad de la flora y vegetación españolas ante el cambio climático. Revista Ecosistemas, 21(3), 1-6.

Gallopín, G. (2003). Sostenibilidad y desarrollo sostenible: un enfoque sistémico. Serie Medio Ambiente y Desarrollo. Santiago, Chile: CEPAL Recuperado de http://repositorio.cepal.org/bitstream/handle/11362/5763/S033120_es.pdf?sequence=1

Gemenne, F. y Reuchlin, P. (2008). Asia central, Migraciones Forzadas. Reino Unido: Universidad de Oxford.

GESAMP. (2001). Planning and management for sustainable coastal aquaculture development (Reports and Studies N. ${ }^{\circ} 68$ ). Recuperado de 
http://www.jodc.go.jp/jodcweb/info/ioc_doc/ GESAMP/Y1818e00.pdf

Hernández, J. R., Ortiz Pérez, M. A., Méndez Linares, A. P. y Gama Campillo, L. (2008). Morfodinámica de la línea de costa del estado de Tabasco, México: tendencias desde la segunda mitad del siglo XX hasta el presente. Investigaciones geográficas, 65, 7-21.

Herrera, R. (2006). La perspectiva teórica en el estudio de las migraciones. Siglo XXI: México.

Herrera, W. (1985). Clima de Costa Rica. San José, Costa Rica: EUNED.

Hoegh-Guldberg, O., Mumby, P. J., Hooten, A. J., Steneck, R. S., Greenfield, P., Gómez, E. y Knowlton, N. (2007). Coral reefs under rapid climate change and ocean acidification. Science, 318(5857), 1737-1742.

Holdridge, L.R. (1967) Life Zone Ecology. Tropical Science.

Hopkins, J.S. (1996). Aquaculture sustainability: avoiding the pitfalls of the green revolution. World Aquaculture, 27(2): 13-15.

Huq, S. y Reid, H. (2007). A vital approach to the threat climate change poses to the poor. International Institute for Environment and Development (IIED). Recuperado de http://pubs.iied. org/pdfs/17005IIED.pdf?

Instituto Costarricense de Turismo (ICT). (2002). Plan General de Desarrollo Turístico Sostenible 2002-2012. San José, Costa Rica. 170 p.

Instituto Metereológico Nacional (IMN). (2008). Boletín meteorológico. Recuperado de https:// www.imn.ac.cr/boletin-meteorologico

IPCC. (2007). Climate Change 2007: Impacts, Adaptation and Vulnerability. Cambridge University Press, Cambridge.

IPCC. (2013). Climate Change 2013: The Physical Science Basis. United Kingdom and New York, NY, USA: Cambridge University Press. Re- cuperado de http://ipcc.ch/report/ar5/wg1/\#. UkYBqtIz08y

Jiménez, C. E. y Suescún, J. I. S. (2011). Los desplazados ambientales más allá del cambio climático. Un debate abierto. Cuadernos Geográfi$\cos , 49,201-215$.

Landesman, L. (1994). Negative impacts of coastal aquaculture development. World Aquaculture 25,12-15.

Lira, L. (2006). Revalorización de la planificación del desarrollo. Santiago, Chile: CEPAL.

Macintosh, D.J. y Phillips, M.J. (1993). Environmental Issues in Shrimp farming.

Ministerio del Ambiente Energía y Telecomunicaciones (MINAET), Instituto Meteorológico Nacional (IMN), Programa de las Naciones Unidas para el Desarrollo (PNUD) y Comité Regional de Recursos Hidráulicos (CRRH). (2008). Clima variabilidad y Cambio Climático en Costa Rica. Recuperado de http://cglobal. imn.ac.cr/Pdf/adaptacion/El\%20clima,\%20variabilidad $\% 20 y \% 20$ cambio $\% 20$ climatico $\% 20$ en\%20CR\%20(version\%20final).pdf

Mora, F. (2014). Políticas de adaptación al cambio climático en zonas costeras alternativas para las comunidades en Costa Rica. Perspectivas FES, 3, 1-8.

Morales, E. (2000). Estudios Sociales 4. San José, Costa Rica: Santillana. 159 p.

Moreno, J. M., De la Rosa, D. y Zazo, C. (2005). Evaluación preliminar de los impactos en España por efecto del cambio climático. Castilla-La Mancha, España.

Peñuelas, J., Sabaté, S., Filella, I. y Gracia, C. (2004). Efectos del cambio climático sobre los ecosistemas terrestres: observación, experimentación y simulación. En Valladares, F (Ed). Ecología del Bosque Mediterráneo en un Mundo Cambiante (425-460). Madrid, España: Ministerio de Medio Ambiente. 
Piedra-Castro, L.; Bravo Chacón, J. y Salazar Herrera, B. (2009). Mamíferos del Refugio Nacional de Vida Silvestre Limoncito, Westfalia, Limón, Costa Rica. Brenesia, 71-72, 21-26.

ProAmbi. (1996). Plan de Manejo para el Refugio Nacional de Vida Silvestre Gandoca- Manzanillo. Vol. I- II Y III. Proyecto de fortalecimiento institucional del SINAC. UCR- ProAmbi/ MINAE, San José, Costa Rica. 360 p.

Quesada. (1997). Struktur und Dynamik eines tropischen Feuchwaldes nach Holznutzung in Costa Rica. Fachbereicht: Universität Gottingen.

Orr, J. C., Fabry, V. J., Aumont, O., Bopp, L., Doney, S. C., Feely, R. A. y Key, R. M. (2005). Anthropogenic ocean acidification over the twenty-first century and its impact on calcifying organisms. Nature, 437(7059), 681-686.

Resilience Alliance. (2002). Resilience. Recuperado de http://www.resalliance.org/index.php/ resilience

Roberts, R.J. y J. F. Muir. (1995). 25 Years of World Aquaculture: Sustainability, a global program. En Reinertsen H. y Haaland H. (Ed). Sustainable Fish Farming (167-181). Rótterdam, Netherlands; Brookfield, Estados Unidos: Balkema.

Rojas, M., Campos, M., Alpízar, E., Bravo-Chacón, J. y Córdoba-Muñoz, R (2003). El cambio climático y los humedales en Centroamérica: Implicaciones de la variación climática para los ecosistemas acuáticos y su manejo en la región. San José, Costa Rica: IUCN.

Romer, K.(2006). ¿Refugiados 'ambientales'?, Migraciones Forzadas. Universidad de Oxford, 25, 61.
Sepúlveda, C. y Ibrahim, A. (2009). Políticas y sistemas de incentivos para el fomento y adopción de buenas prácticas agrícolas: como una medida de adaptación al cambio climático en América Central. CATIE.

Sharp, G.D. (2004). Cambio climático y pesquerías regionales en el futuro: análisis en colaboración. Recuperado de http://www.fao.org/docrep/007/y5028s/y5028s00.htm

Thomas, J. R. y Nelson, J. K. (2007). Métodos de investigación en actividad física. Barcelona, España: Paidotribo.

Vallega, A. (1999). Fundamentals of Integrated Coastal Management. Dordrecht, Netherlands Kluwer Academic Publishers.

Walker, B., Carpenter, S., Anderies, J., Abel N., Cumming G., Janssen, M., Lebel, L., Norberg, J., Peterson, G.D. y Pritchard, R. (2002). Resilience Management in socioecological systems: a working hypothesis for a participatory approach. Conservation Ecology, 6(1), 1-17.

Weitzner, V. y Fonseca, M. (2000). Cahuita, del conflicto a la colaboración en cultivar la paz: Conflicto y Colaboración en el Manejo de Recursos Naturales. Ed Daniel Bucles, Canadá.

Yáñez-Arancibia, A., Twilley, R. R. y Lara-Domínguez, A. L. (2016). Los ecosistemas de manglar frente al cambio climático global. Madera y Bosques, 4(2), 3-19.

Recibido: 01 de octubre de 2018 Aceptado: 23 de noviembre de 2018 